\title{
The Beliefs and Attitudes of Cypriot Physical Therapists Regarding the Use of TENS in Pain Management
}

\author{
Sophia Tzioni ${ }^{2}$ and Dimitrios Stasinopoulos ${ }^{1 *}$ \\ ${ }^{1}$ Department of Health Sciences, School of Sciences, European University Cyprus, Cyprus \\ ${ }^{2}$ Director of Cyprus Musculoskeletal and Sports Trauma Research Centre (CYMUSTREC), Cyprus \\ *Corresponding author: Dimitrios Stasinopoulos, Department of Health Sciences, School of Sciences, European University Cyprus, \\ Cyprus
}

\section{ARTICLE INFO}

Received: 豐 December 03, 2019

Published: December 10, 2019

Citation: Sophia Tzioni, Dimitrios Stasinopoulos. The Beliefs and Attitudes of Cypriot Physical Therapists Regarding the Use of TENS in Pain Management. Biomed J Sci \& Tech Res 23(4)-2019. BJSTR. MS.ID.003948.

Keywords: TENS; Pain; Cyprus; Physiotherapist; Attitudes; Beliefs

Abbreviatations: IASP: International Association for the Study of Pain; CNS: Central Nervous System; TENS: Transcutaneous Electrical Nerve Stimulation; RMV: Rostral Ventromedial Medulla; PAG: Periaqueductal Gray; SC: Spinal Cord

\section{ABSTRACT}

Background: Pain is the main cause of a patient's visit to the physiotherapist. TENS is a non-invasive analgesic technique that is worldwide used. The physiotherapist's role is very important in the treatment of the patient and many studies support the effectiveness of TENS to improve the pain levels. However, there is still a difference in its use by therapists and researchers.

Objectives: The aim is to collect information about the beliefs and attitudes of Cypriot physiotherapists about the analgesic effect of TENS.

Methods: The questionnaire was obtained from a previous study in India and sent via an online platform to Cypriot physiotherapists. The statistical analysis was done by SPSS.

Results: 113 responses were received, where most physiotherapists reported TENS as a successful way of treatment. TENS seemed to be used more often in acute and intense pain, as well as in musculoskeletal pain, compared to pain due cancer. Almost all physiotherapists reported that their patients benefit to some extent from the TENS and most would suggest it as a home remedy. TENS is a popular prescription, while most Cypriots request or have already used it. 58.4\% of Cypriot physiotherapists believe it is cost-effective while $28.3 \%$ said that they did not know. Most of them provide devices similar to TENS. There was a significant correlation of the TENS use in acute pain with its use in musculoskeletal/orthopaedic conditions $(\mathrm{p}=0.000)$, and a significant correlation of the TENS efficacy with the TENS use frequency for pain treatment $(p=0.000)$.

Conclusion: TENS is mostly used to reduce pain and is considered effective. Their views are largely similar to physiotherapists from other countries. More high-quality research is needed to explore and compare their views with research results.

\section{Introduction}

Pain

Based on the International Association for the Study of Pain (IASP) pain is "an unpleasant sensory and emotional experience associated with actual or potential tissue damage or described in terms of such damage"[1]. Pain is a protective tool, but if it loses its adaptive property it can get converted to a pathological issue. Chronic pain has been accepted as a pathology and a disorganization of the nervous system. It can be caused by genetic predisposition, central sensitization mechanisms and other factors [2]. John J. Bonica (1953) suggested that chronic pain sensation no longer serves a useful purpose and has a psychological and somatic affect [2].

\section{Pain classification}

Pain can be classified in many ways. The most usual ways are according the anatomy, intensity, duration and pathophysiology $[3,4]$. The pain sensation is individual and subjective [5]. 


\section{Epidemiology of Pain}

Based on an international research in 2014, at least $10 \%$ of the population is affected by chronic pain and 1 out of 10 people will be additionally diagnosed every year [2]. In European (EU) 1 out of 5 adults is affected by chronic pain, mostly women, older age people and people with lower socioeconomic status [6]. However, men are less willing to report their pain in comparison with women [7]. Children of people with chronic pain are in greater risk of developing chronic pain [8]. In USA the cost of therapy for people with chronic pain is estimated at \$560-635 billion per year [4] and in EU at $200 €$ billion [8]. Musculoskeletal pain is significant more often now than 40 years ago and is related with obesity. Shoulder, back and widespread pain have increased about 2-4 times, in the past 40 years [9] (Figure 1).

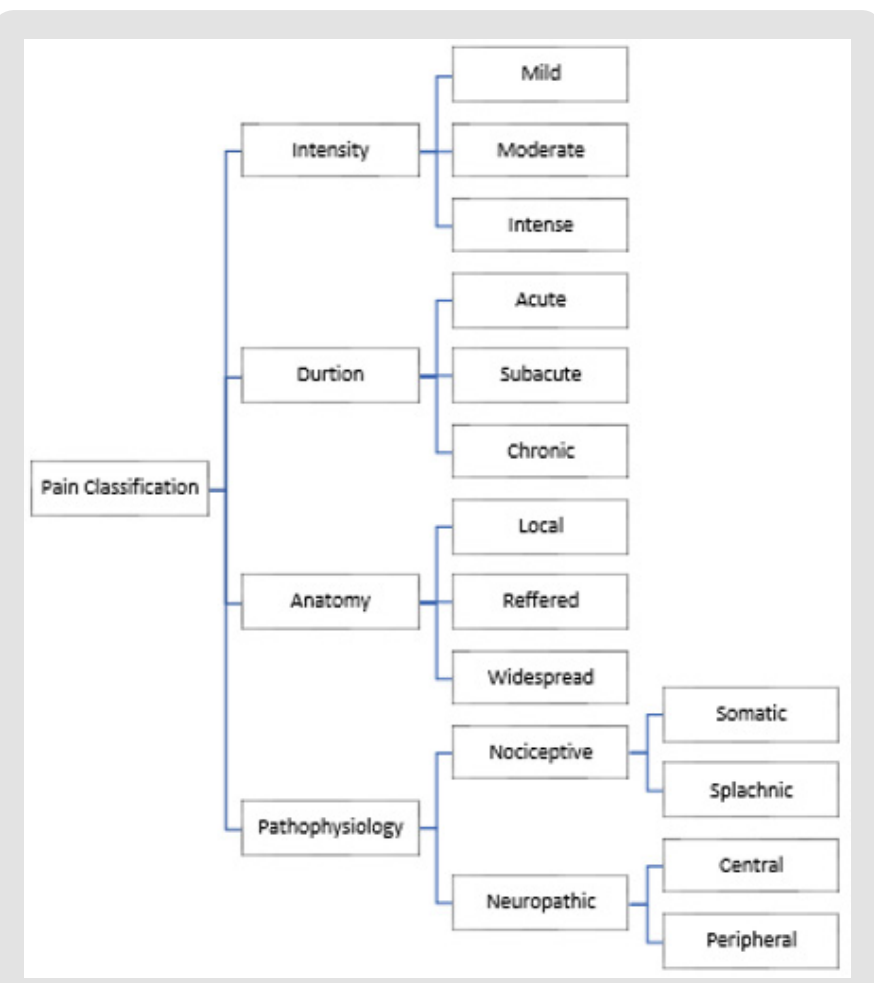

Figure 1: Classification of Pain.

\section{Pain Mechanism}

An injury initiates a series of processes to resolve the trauma. Cytokines and neuropeptides are released from the nerve endings and start the peripheral sensitization by initiating the inflammatory process $[10,11]$. The body and brain perceive the pain through nociception, which is a neural process of coding and processing noxious stimuli [5]. The nociceptors perceive and transfer the noxious stimuli from peripheral to central to the highest structures [12] after it gets transformed at the nerve endings to an electrical signal [13]. The nociceptors stimulate and release glutamine as their main neurotransmitter and other substances (substance P, calcitonin, somatostatin) to help the signal transmission at the synapsis [5]. Pain transmits through an action potential which has encoded all the information about the noxious stimuli $[4,14]$.
The action potential travels through the dorsal horn and the spinothalamic tract to the brainstem, cerebellum and thalamus [5]. In the Central Nervous System (CNS) the coded information is controlled by the cortical centers and the brainstem, which can act as stimulants or inhibitors, form the emotional and sensory parts of pain and initiates appropriate to inhibit pain and start the healing process $[4,15]$ (Image 1). A pathological condition of the CNS is the central sensitization, caused by nonstop release of neurotransmitters and results to continuous stimulatory action of the dorsal horn and CNS [11]. A potential reason for that can be the imbalance between the inhibitory and stimulatory mechanism of pain, with enhanced central stimulation and/or reduced central inhibition [4].Another pathological situation is the fear avoidance behaviour. After experiencing pain while performing a certain movement the body inhibits it to avoid the pain occurrence [10]. This can result to a creation of a pathological movement pattern with alteration of the muscle condition because of inhibiting the agonists of the painful motion and over stimulating the muscles of the alternated pathological movement pattern [4].

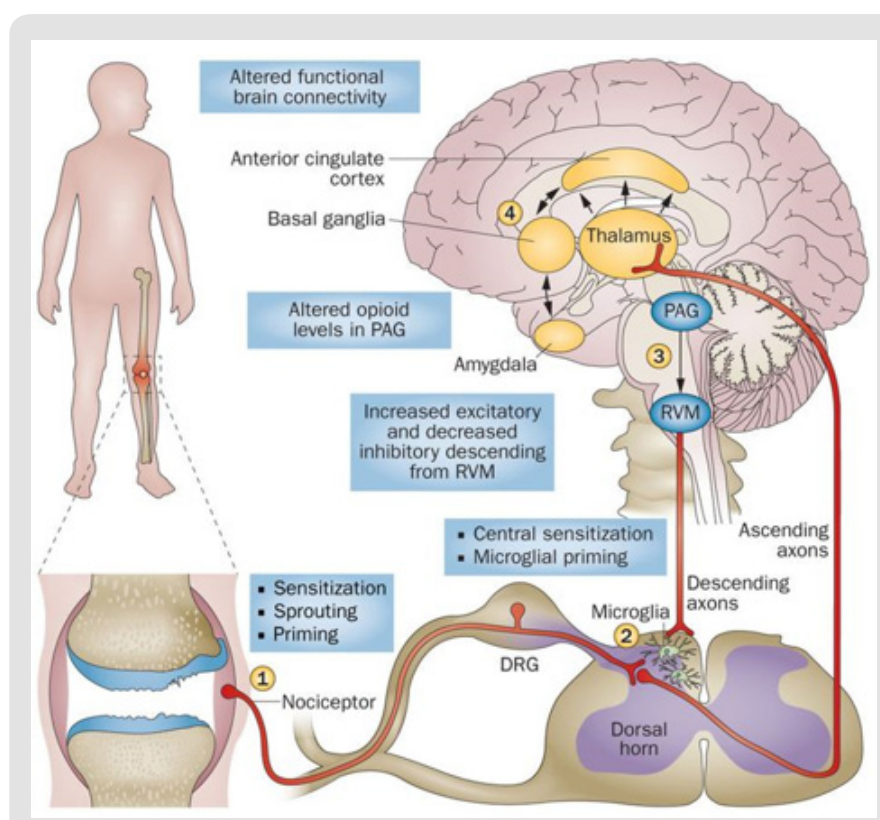

Image 1: Pain perception pathway [65].

\section{TENS}

Transcutaneous Electrical Nerve Stimulation (TENS) by definition is anything that delivers electricity across the intact surface of the skin to activate underlying nerves [16]. The TENS devise is a non-invasive technique of transferring non painful pulsed electric current across the skin and is characterised by low cost and usage simplicity [17].

\section{Effects of TENS}

TENS has been used for pain relieve, to improve decreased mobility, increase function, improve quality of life, increase the tolerance in pain, increase heart rate and decrease arterial blood pressure, change tissue temperature, produces vasodilatation, 
helps tissue repair, accelerates scar healing after amputation and restore secondary movement alteration when the primary issue is avoids further amputations diabetes [18,21]. It can also help pain [22] (Table 1).

Table 1: Indications and Contraintications of TENS use [21-23].

\begin{tabular}{|c|c|}
\hline \multicolumn{2}{|c|}{ Indications } \\
\hline Acute disorders & Trigeminal neuralgia \\
\hline Post-surgical pain & Imaginary pain \\
\hline Dysmenorrhea & Diabetic neuropathies \\
\hline Oral pain & Nerve entrapment \\
\hline Dental procedures & Reflex sympathetic dystrophy \\
\hline Acute low back pain & Causalgia \\
\hline Localized muscle pain & Cancer-related pain \\
\hline Post-herpetic neuralgia & Reason \\
\hline Contraindications & Possible irritation of the carotid artery and laryngeal nerve \\
\hline Anterior neck area & Possible increase of blood pressure in the eye \\
\hline Around eyes & Possible alteration of heart beat and function of intercostal muscles \\
\hline Anterior or posterior sternum & Possible alteration of the defibrillator rhythm \\
\hline People with cardioverter-defibrillator & Unknown possible effects \\
\hline Pregnancy & Unknown possible effects \\
\hline Epilepsy & Unable to follow instructions \\
\hline People with reduced perception & Possible skin irritation due to excessive intensity \\
\hline Alternated sensation & \\
\hline
\end{tabular}

\section{Characteristics of TENS Device}

Different TENS types activate different type of neurons and neuroreceptors and are producing different mechanism of

Table 2: Indications

\begin{tabular}{|c|c|c|c|c|c|}
\hline Type & Frequency & Intensity & Pulse duration & Sensitized fibers & Sensation \\
\hline Conventional & $80-120 \mathrm{~Hz}$ & Low & $50-100 \mu \mathrm{s}$ & $\begin{array}{c}\text { Large diameter, low threshold afferent neurons } \\
(\mathrm{A} \beta)\end{array}$ & $\begin{array}{c}\text { Strong, non painful paraes- } \\
\text { thesia }\end{array}$ \\
\hline Acupuncture like & $2-4 \mathrm{~Hz}$ & High & $100-200 \mu \mathrm{s}$ & $\begin{array}{c}\text { Small diameter, high threshold afferent neurons } \\
(\mathrm{A} \delta \text { ) }\end{array}$ & Non painful muscle spasms \\
\hline Intense & $>12 \mathrm{~Hz}$ & High & $>200 \mu \mathrm{s}$ & $\begin{array}{c}\text { Small diameter, high threshold afferent neurons } \\
(\mathrm{A} \delta \text { ) }\end{array}$ & Strong, tolerable paresthesia \\
\hline Type & Frequency & Intensity & Pulse duration & Sensitized fibers & Sensation \\
\hline
\end{tabular}

\section{Analgesia and Analgesic Effect of TENS}

Melzack and Wall (1965) created a theoretical model of the inhibition of noxious stimulus, named gate control theory [25]. According to this theory after an injury there is immediate activation of small diameter afferent neurons, the signal reaches gray matter (GM) and opens the gate and transmits the stimuli to the brain. Reflexively the body activates large diameter afferent neurons and inhibits the painful stimuli at the GM to decrease pain [25]. Central analgesia is produced by the descending analgesic mechanism, which starts at the rostral ventromedial medulla (RMV) and the periaqueductal gray (PAG) of the midbrain [26]. Another analgesic process is through the sympathetic mechanism which reduce the sympathetic tone [27]. analgesia. There are is no correlation between electrode placement and effectiveness in patients with chronic pain [24] (Table 2).
The exact mechanism of action of the TENS is not way completely understood and there are many theories which explain it [27]. Its activity is mainly through the function of neurochemicals such as opioids, serotonin, acetylcholine, noradrenaline and gammaaminobutyric acid and activation of the central and peripheral analgesic mechanisms [28]. Research has shown an increase in GM activity and subsequent release of endorphins and endogenous opioids in people with musculoskeletal pain [18]. In studies the TENS mechanism of action is divided into spinal, supraspinal and peripheral $[23,29]$. There is activation of the central mechanism, RMV and PAG [23,29,30]. Supraspinal, at the RMV and spinal cord (SC) there is irritation of the opioid receptors which can have inhibitory or stimulant effect and can reduce or increase the function of the neurons at the GM [31]. 


\section{Materials and Methods}

\section{Questionnaire Design}

The questionnaire used in the present study was taken from the previous survey conducted in India (Appendix 1) [32]. The questionnaire consisted of 11 multiple-choice questions and has been translated into Greek. All questions included were required to be answered while only one sub-question was non-obligatory and included a non-obligatory open-ended field. In the multiplechoice questions, respondents could only choose one answer. The questions asked by the authors of the questionnaire concern the use of TENS by Physiotherapists on pain-management. The present study had interest to Cypriot Physiotherapists. The questions were aimed at obtaining information on the frequency of treatment, the frequency of treatment of pain with/without the device, and the frequency depending on the duration and pathology of the pain. It was also examining whether patients benefit from the use of TENS, whether the clinician recommends home therapy with the device and whether doctors suggest physiotherapist TENS use, if patients seek electrotherapy as a means of relief and if used before physiotherapy. Also, it was looking on the opinion of TENS cost-effectiveness and whether they use other TENS like devises (Appendix 2). The questionnaire has not been validated to the best of our knowledge. The researchers who created it had 12 questions, with the latter asking for the personal and contact information. Our research was anonymous and no information was requested.

\section{Questionnaire Sharing and Collection}

The questionnaire was sent to more than 840 Cypriot physiotherapists working in the private and public sector via an electronic platform by email and through the social media. Minimum number of responses was set to 55. The collection took 4 months. Physiotherapist were asked twice to complete the questionnaire, 3 months apart.

\section{Statistical Analysis and Data Processing}

The study examined the percentages of each response using an online platform and the correlation of the answers to the questions using the SPSS. Due to the existence of many cells in different categories of responses, the $\mathrm{x} 2$ control could not be used correctly, so instead of chi-square we used the decision tree. Statistical significance was set at $\mathrm{p} \leq 0.05$.

\section{Ethical Issues}

Prior to the questionnaire, respondents received anintroductory message stating that participation in the survey was voluntary and withdraw could happen at any time without giving any explanation. It was also stated that the investigation was anonymous and that no personal information would be requested. The participants had no benefit from their participation and that the results would be likely to be published in physiotherapy journals and online. Participants had contact information of the researcher if more information was needed.

\section{Results}

Of the 113 people who responded to the questionnaire, 81.4\% responded that they treat pain in their clinical practice very often, $15 \%$ often, $3.5 \%$, rarely (Figure 2 ). All but two responded that they use TENS to treat patients with pain, $38.1 \%$ of them very often, $37.2 \%$ often, $8.8 \%$ occasionally, $14.2 \%$ rarely and $1.8 \%$ never (Figure 3). The third question included sub-questions and asked physiotherapists how often they use the TENS device for each type of pain. Pain duration was divided into acute, subacute and chronic. In acute pain $42.5 \%$ responded very often, $27.4 \%$ often, $22.1 \%$ occasionally, $5.3 \%$ rarely, while $2.7 \%$ never. In subacute pain $24.8 \%$ said very often, $35.4 \%$ often, $24.8 \%$ occasionally, $8.8 \%$ rarely and $6.2 \%$ never. For chronic pain $23.9 \%$ very often, $20.4 \%$ often, $30.1 \%$ occasionally, $17.7 \%$ rarely and $8 \%$ never. Pain intensity was divided in mild $(<2 / 10)$, mild-moderate $(2-4 / 10)$, moderate $(4-6 / 10)$, moderate-severe (6-8/10) and severe $(>8 / 10)$. For the treatment of mild pain $11.5 \%$ use TENS very often, $16.8 \%$ often, $36.3 \%$ occasionally, $17.7 \%$ rarely, $17.7 \%$ never. For mild-moderate pain $12.4 \%$ very often, $21.2 \%$ often, $36.3 \%$ occasionally, $17.7 \%$ rarely and $12.4 \%$ never. For moderate pain $14.2 \%$ very often, $30.1 \%$ often, $34.5 \%$ occasionally, $12.4 \%$ rarely and $8.8 \%$ never. For moderatesevere pain $26.5 \%$ very often, $31 \%$ often, $32.7 \%$ occasionally, $7.1 \%$ rarely and $2.7 \%$ never.

1. How often do you treat pain in your clinical practice?

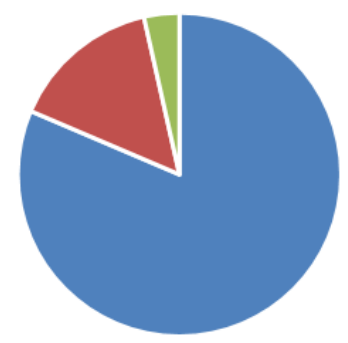

- Very often - Often = Seldom

Figure 2: Frequency of pain treatment in clinical practice.

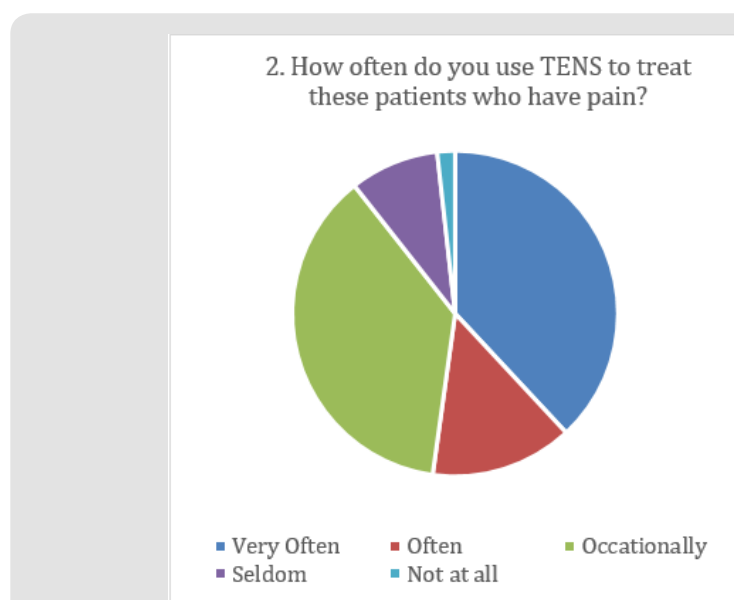

Figure 3: Frequency of TENS use for analgesia. 
For severe pain $40.7 \%$ very often, $25.7 \%$ often, $22.1 \%$ occasionally, 6.2\% rarely and 5.3\% never (Figure 4). Aetiology of pain was divided in various diseases musculoskeletal and/ or orthopaedic disorders, neuropathies and/or neuralgia, postsurgery and cancer. For the treatment of musculoskeletalorthopaedic pain $52.2 \%$ use TENS very often, $25.7 \%$ often, $15 \%$ occasionally, $6.2 \%$ rarely, < $1 \%$ never. For neuropathic painneuralgia, $13.3 \%$ very often, $21.2 \%$ often, $32.7 \%$ occasionally, $14.2 \%$ rarely and $18.6 \%$ never. For post-surgery pain $15.9 \%$ very often, $27.4 \%$ often, $31.9 \%$ occasionally, $14.2 \%$ rarely and $10.6 \%$ never. For pain in cancer patients, $7.1 \%$ very often, $>1 \%$ often,
$18.6 \%$ occasionally, $15 \%$ rarely and $58.4 \%$ never. The last nonobligatory questionnaire investigated how often TENS is used for other conditions. It was answered by 22 physiotherapists, $2.7 \%$ of them said often, $8 \%$ occasionally, 3.5\% rarely and 5.3\% never (Figure 6). In the non-obligatory explanatory field, the six answers that obtained at once are «relaxation», «muscle strain», «interstitial cystitis», «herpes zoster», "generally do not use TENS» and «no». Some benefit to their patients during and/or after the use of TENS observed the $56.6 \%$ of the therapist, $41.6 \%$ observed little benefit and $1.8 \%$ no benefit (Figure 5).

\section{For which types of pain do you use TENS?}

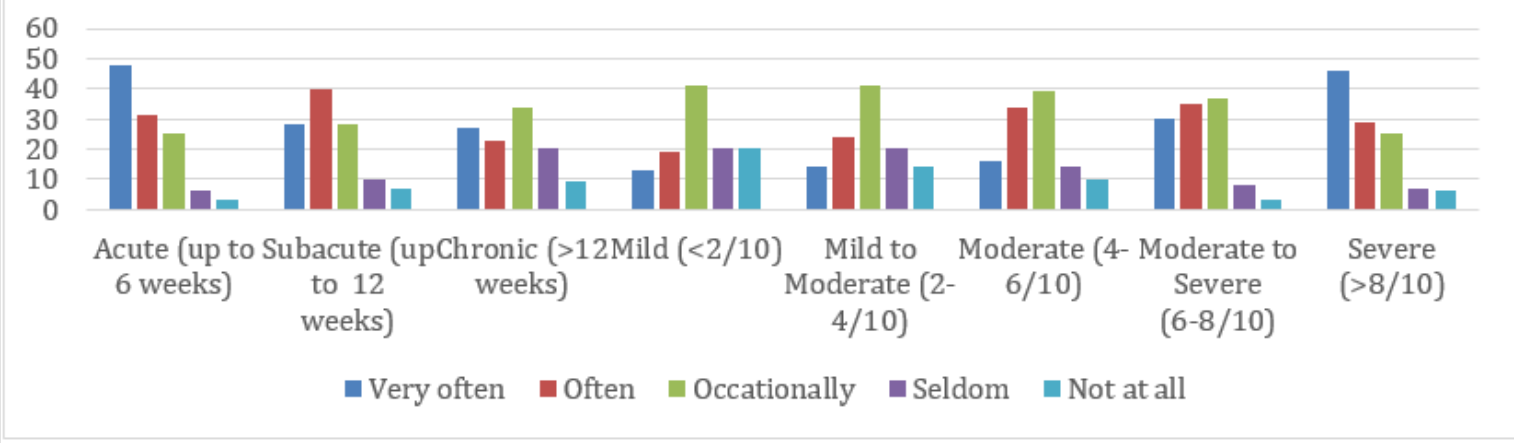

Figure 4: Frequency of use of TENS for the treatment of pain depending on its duration and intensity.

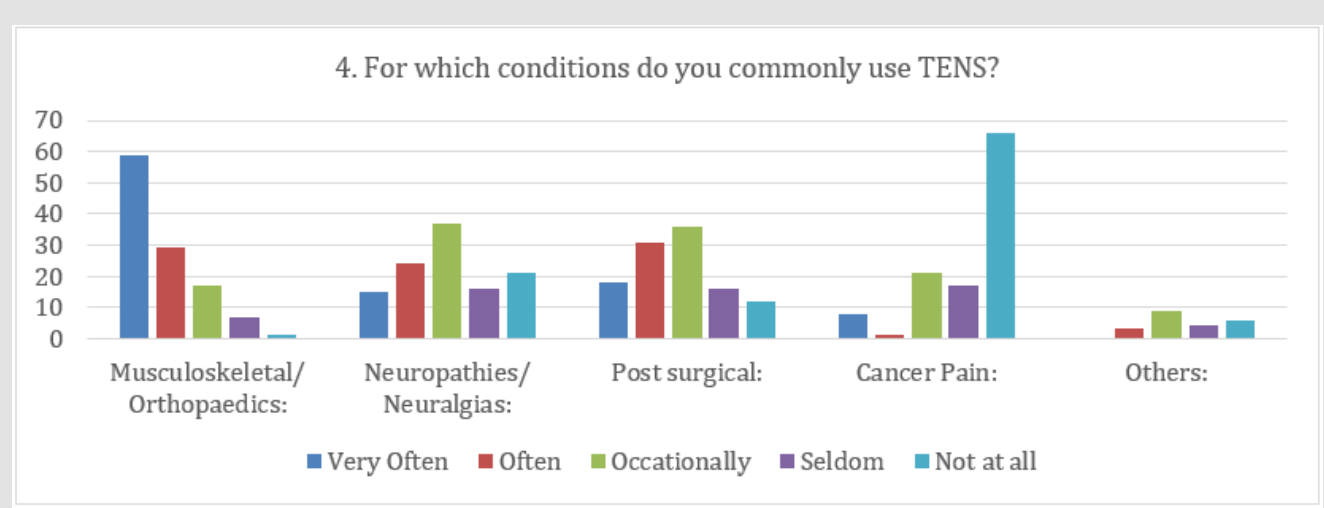

Figure 5: Frequency of use of TENS for the treatment of pain depending on the cause of the pain.

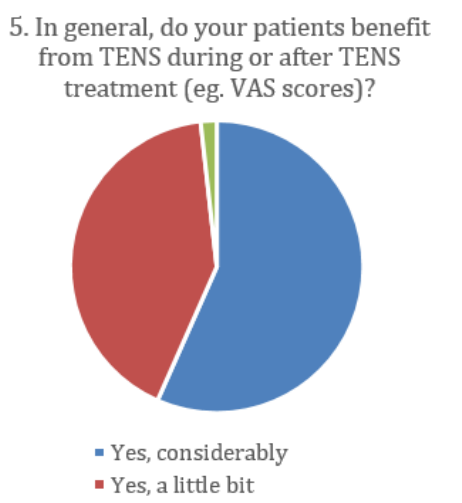

Figure 6: Benefits of TENS during or after treatment. 
Home therapy with TENS would recommend $44.2 \%$ of physiotherapists, $31.9 \%$ would recommend occasionally and 23.9\% would not recommend it (Figure 7). TENS prescription for analgesia by physicians was observed by $89.8 \%$ of physiotherapists, of them $54 \%$ quite often, $35.4 \%$ not often enough and $10.6 \%$ had never been consulted by doctors for TENS use (Figure 8). Most physiotherapists $(71.7 \%)$ had been asked for TENS use by patients, while $28.3 \%$ had not been asked (Figure 9). Patients were using TENS before going to receive pain treatment from physiotherapist responded $54.9 \%$ and have not responded $45.1 \%$ (Figure 10). As cost-effective compare to other devises assess the TENS the $58.4 \%$, $13.3 \%$ believed it was not, and $28.3 \%$ responded that they did not know (Figure 11). Other TENS-like are provided by the $44.2 \%$ of physiotherapists, $34.5 \%$ responded occasionally and $21.2 \%$ did not provide (Figure 12). During the statistical analysis, the beliefs and tendencies of the Cyprus physiotherapists were investigated. In correlating the efficacy of TENS in pain with other questions, a significant relationship was found between the use of TENS in acute pain and its use in musculoskeletal-orthopaedic diseases (Figure 13). Physiotherapists who use it more frequently in acute pain, use it more often in musculoskeletal-orthopaedic diseases, while those who use it less frequently in the treatment of acute pain use it more occasionally ( $p$-value $=0.000$, chi-square $=28.617, \mathrm{df}=4$ ).

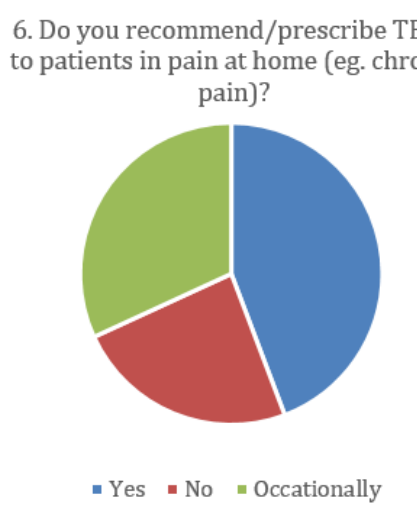

Figure 7: View of Cypriot Physiotherapists whether proposed use of TENS at home by patients.

\section{Does your referring physician (eg} orthopaedician) advise you to use TENS for pain relief?

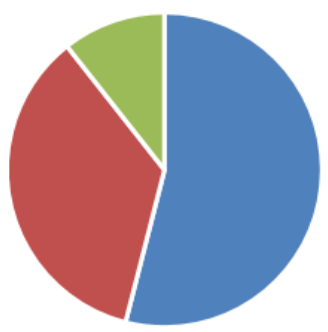

- Yes, quite ofte - Yes, but not very often = Never

Figure 8: Frequency of referrals from physicians for TENS use for pain relief.
8. Have any of your patients ever requested TENS treatment from you?

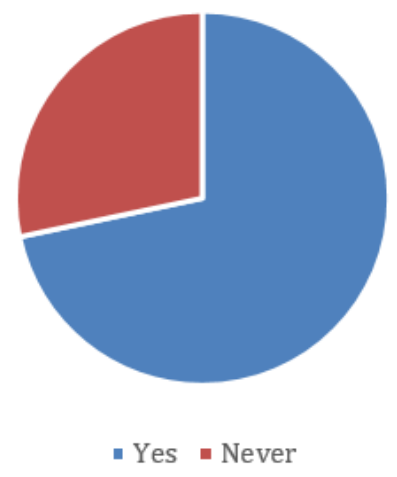

Figure 9: Investigating whether patients require TENS as a means of treatment.

\section{Have any of your patients said that} they used TENS before they came to receive pain treatment from you?

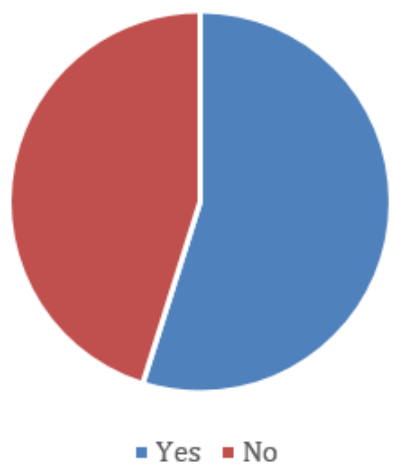

Figure 10: Investigation of whether patients use TENS at home before physiotherapy.

10. Do you think TENS treatment is
cost effective compared with other
treatments (eg physical medicine, drug treatments (eg physical
therapy)?

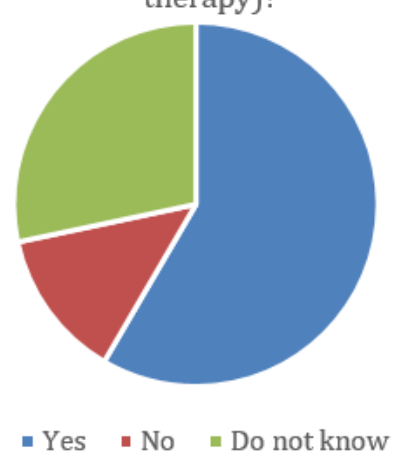

Figure 11: Cypriot Physiotherapists' View of TENS CostEffectiveness Compared to Other Treatments. 
11. Do you offer any other types of

TENS-like devices such as

interferential therapy to treat pain for your patients?

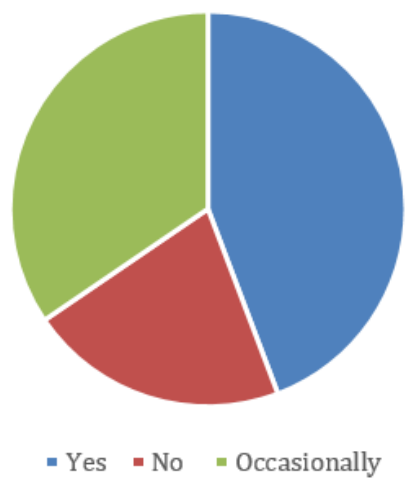

Figure 12: Investigation of the supply of TENS like devices by Cypriot physiotherapists for the treatment of pain.

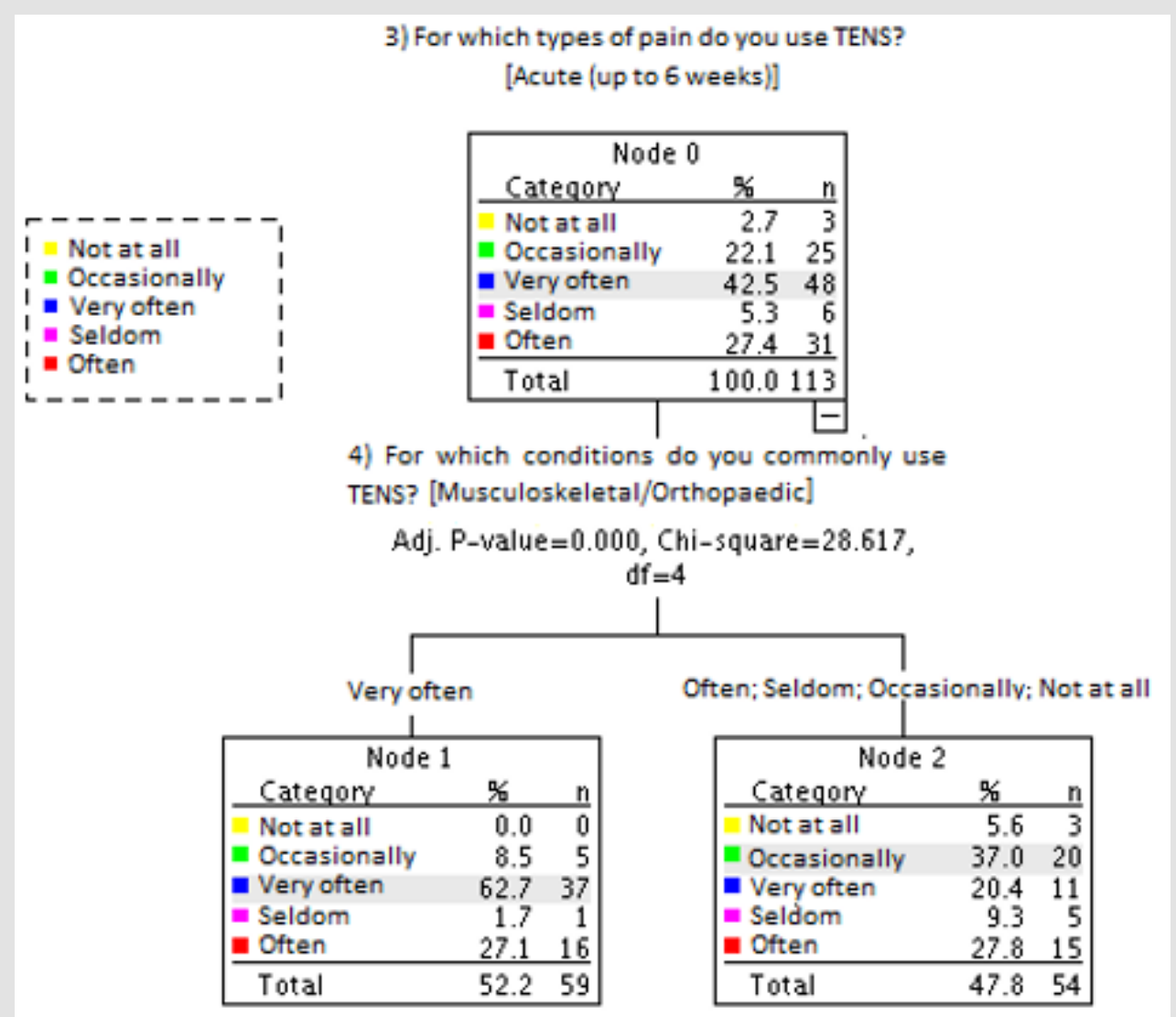

Figure 13: Relationship between the use of TENS by Cypriot physiotherapists for the treatment of acute pain and the frequency of TENS use in musculoskeletal / orthopedic diseases.

In correlating the efficacy of TENS in reducing pain with the frequency of using TENS for pain treatment (Figure 14), it appeared that physiotherapists who their patients benefited enough from TENS, use TENS very often or often to treat patients with pain. On the other hand, whose patients benefit little from TENS, appeared to use TENS more occasionally, rarely or not at all ( $p$-value $=0.000$, chi-square $=23.330, \mathrm{df}=2$ ). No other correlation was found with the efficacy of TENS and the other questions. The other correlations found to have significant effects are listed in Appendix 3, but do not lead to any significant findings on the beliefs and attitudes of Cypriot physiotherapists. 


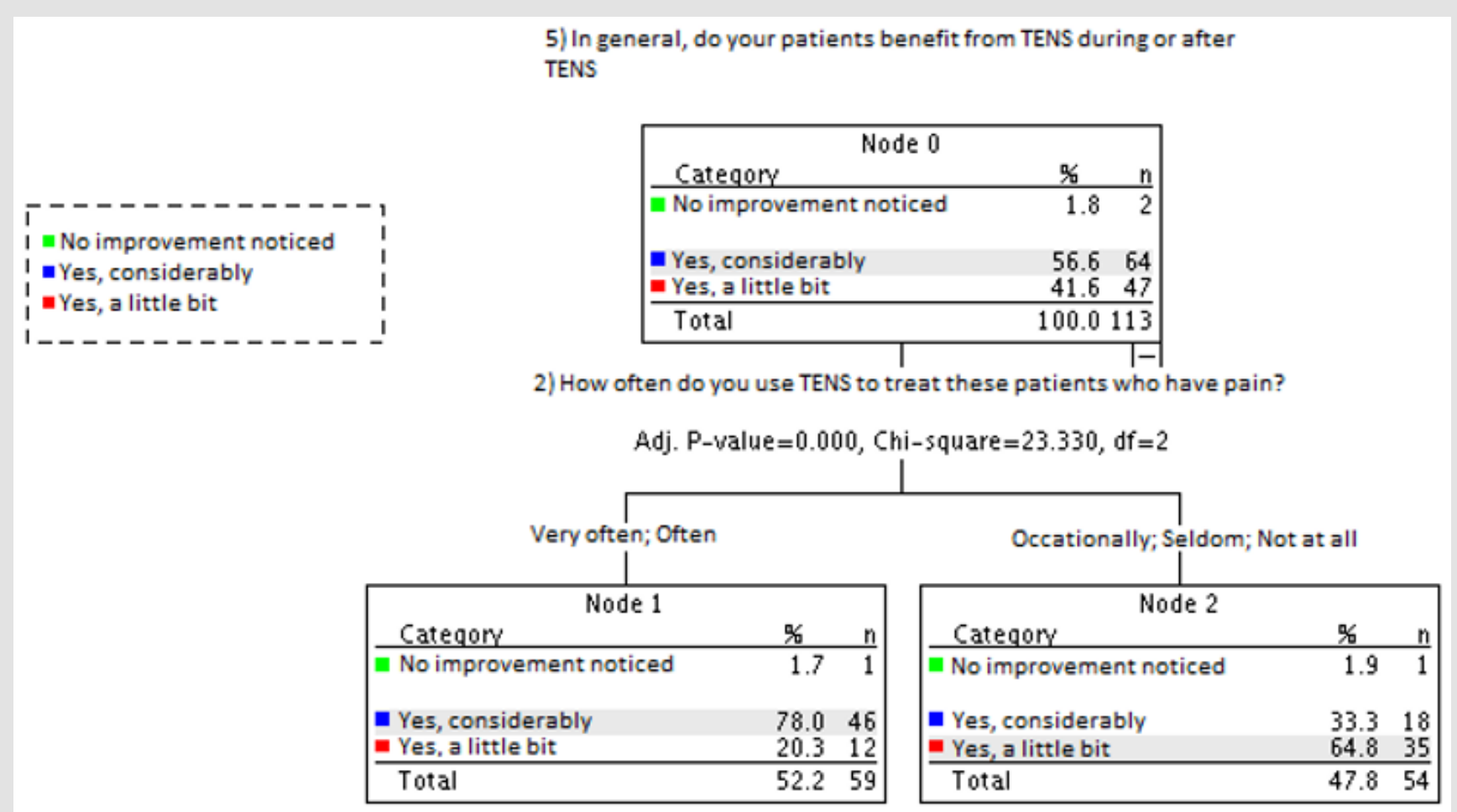

Figure 14: Relationship between the efficacy of TENS in reducing pain by Cypriot physiotherapists with the frequency of using TENS for pain treatment.

\section{Discussion}

\section{General Discussion}

Pain is one of the most common reasons for visiting physiotherapists to reduce their pain and functional impairments [33]. Physiotherapy is an essential component of chronic pain management. Studies show that physiotherapists can use a wide range of practice to guide and support people with chronic pain [34]. We received 113 responses which are satisfactory as 1040 were registered in the Cyprus Physiotherapists Association by 2018 , according to the latest available published version. The largest percentage of Cypriot physiotherapists reported healing pain in his clinical practice. A study by the European Union (EU) shows that the expenditure of the Republic of Cyprus on the health of Cypriots is lower than in most countries of the EU. In 2015, Cyprus spent 1592 euros per person on its medical care, compared to the EU average of 2797 euros. However, Cyprus is currently undergoing a transitional phase in the health sector as the implementation of a general health system has begun. Physiotherapists are not yet part of the general health system, with admission likely to begin in June 2020 [35]. TENS seems to be used extensively by Cypriot physiotherapists. Compared to Australia, it seems to be used more in Cyprus, as in Australia around 10\% use it at least once a day, and about 30\% do not [36]. In Israel, a similar survey with Australia also found a large difference, with $66 \%$ responding that they use it at least once a day and $16 \%$ not using it at all [37]. In Japan, with the same research protocol, only $8 \%$ reported using TENS daily, while
$46 \%$ responded that they did not use it at all [38]. In Sri Lanka and India, surveys conducted followed the same protocol as the present study [32,39]. A survey in Sri Lanka found that 9.5\% managed pain with TENS very often while no one responded that they did not use it at all [39] In the Indian study, the results showed that $80 \%$ of physiotherapists use TENS often or very often, however, it is not clear the percentage of very often and often [32]. In USA $72.4 \%$ of physiotherapists reported using the TENS device in their clinical practice, but the study did not investigate the frequency of use of the device [40]. The use of TENS in the UK in Australia and Ireland appears to have increased from 1990 to 2000; however, its use has decreased by $30 \%$ between 2000 and 2009 [38]. The difference in the beliefs of the physiotherapists can be due to their country of origin or perhaps the country in which they are educated. In Australia the use of TENS for pain relief is much lower than in Asian countries. Although Cyprus is politically in the EU, geographically is closer to Asia and is possibly influenced by eastern countries. A study in the United Kingdom found a significant difference between lack of sun, low temperature and pain, but this is not fully proven [8].

TENS has been shown to help in chronic and acute pain without significant differences between them, but with better results in both compared to placebo [41]. Cochrane's reviews of chronic pain in various diseases and situations lead to methodological weaknesses of randomized controlled trials [42]. In another Cochrane review to evaluate TENS as a monotherapy for acute pain reduction 
in adults, the researchers were unable to extract data from the research due to weaknesses in their methodologies [32]. Cypriot physiotherapists use TENS more in acute pain than in subacute and chronic pain. As the duration of the pain increases, it appears that Cypriots are reducing the use of TENS. The same was observed in the Indian study [39]. Sri Lankan's research opposed to Cyprus and India as shows that the use of TENS is increasing as the duration of pain increases [43].

Patients with moderate to severe chronic pain have been estimated to lose an average of 8 days of their work over a sixmonth period, with $22 \%$ losing at least 10 days [44,45]. Studies have shown that lack of sleep, psychological status, socioeconomic status are some of the factors that can make patients' pain worse [46]. Not many studies exist comparing the efficacy of TENS between different pain intensities. A pilot research has shown that TENS has significant effects on pain reduction in patients with severe pain but not in patients with moderate pain [32]. Physiotherapists in Cyprus are increasingly using the TENS device to reduce pain in patients with more severe pain. Figure 4 shows that the frequency of its use increases with increasing intensity. In the Indian study, there is no such a significant change, however, no statistical process has been conducted to investigate this [39]. In research in Sri Lanka, therapists use TENS more frequently as the pain of patients increases, while in mild pain $30 \%$ of physiotherapists do not use TENS [43].

Patients' satisfaction with TENS treatment appeared to depend on the source of the pain. Those who had soft tissue or musculoskeletal pathology had better results with TENS use [18]. Musculoskeletal pain is one of the most common causes of dysfunction, with upper extremity pain occurring approximately $18.6 \%$ to $31 \%$ in adults each month [47]. Osteoarthritis is the most common form of joint pain and is usually local but may also have a reported pain. In the joints there are special type $C$ receptors which do not respond to harmful mechanical stimuli but only when there is inflammation [11]. A study in mice found a different response to experimental pain between the joints and muscles [48]. Joints and tendons have also been found to be more sensitive to experimental pain than muscles [11]. The results of the studies contradict in the efficacy of TENS in arthritic knee pain $[47,49,50]$. In India $68 \%$ of physiotherapists and $60 \%$ of physiotherapists in Sri Lanka reported that they use TENS frequently or very often to reduce musculoskeletal pain [51]. In Great Britain and Hong Kong, physiotherapists believe that TENS is effective in reducing pain in acute musculoskeletal disorders (mean: 5.56/8, SD=1.68; 6.26/8, $\mathrm{SD}=1.66$ respectively) and chronic musculoskeletal disorders (mean: 5.40/8, SD=1.87; 6.46/8, SD=1.71 respectively) [52]. In Cyprus, physiotherapists responded that they use TENS devices in musculoskeletal and orthopedic diseases to reduce pain more than any other condition. It is noteworthy that almost $80 \%$ of physiotherapists in Cyprus said they use it often or very often while only one physiotherapist replied that it does not use it at all for the treatment of musculoskeletal disorders. Compared to Sri Lanka and India, it appears that Cypriots use it about 15\% more. Although there is no data on the epidemiology of musculoskeletal disorders in Cyprus, we can assumethat is high due to the high frequency of use of TENS in these diseases.

A systematic review and meta-analysis in patient with spasticity had strong evidence for TENS effectiveness in spasticity reduction when placed above the nerve or muscle body [53]. However, a Cohort's study in patients with neuropathic pain did not conclude for TENS analgetic effectiveness due to inadequate data, different TENS impamentation and poor quality of surveys [54]. In UK, 70\% of physiotherapists responded in a survey in 1999 that they did not use TENS devices for the treatment of neurological disorders [38], whereas the 2009 survey that they believe TENS is effective for improving pain due to neurogenic diseases (mean: 6.15/8, $\mathrm{SD}=1.79$ ). Hong Kong physiotherapists also believe that TENS is effective in reducing neurogenic pain (mean:6.16/8, $\mathrm{SD}=1.60$ ) [52]. In India, $76 \%$ use TENS often or very often in neuropathies and neuralgia and no one responded that they did not use it, while in Sri Lanka $79.1 \%$ used often or very often and $6 \%$ never [32,39]. In this study, the majority (32.7\%) of Cypriot physiotherapists responded that they use it occasionally, while $18.6 \%$ responded never.

TENS has been found to significantly reduce the pain and need for morphine intake within 24 hours of surgery and to assist in the functional recovery after knee arthroplasty [55]. A systematic review of 29 studies found that there was an analgesic effect following the use of TENS in patients with acute postoperative pain [56]. Another systematic review (2012) in postoperative pain showed a reduction in pain after using conventional TENS and acupuncture like TENS $(n=1350)$. The use of the two types of TENS outweighed the use of placebo with a pain reduction of $26.5 \%$ (mean of all studies) $[57,58]$. TENS has also been shown to increase vascular responsiveness and blood circulation. Experimental studies have had a positive effect on wound healing, and TENS has been shown to reduce ischemic complications after surgery [21]. In India 52\% use TENS frequently or very often to reduce post-operative pain while $4 \%$ do not [32]. In Sri Lanka, about $20 \%$ use it post-surgergicly often or very often, $16.4 \%$ at all, and the highest proportion of physiotherapists in Sri Lanka, approximately 63\%, use it occasionally or rarely [39]. In Cyprus, almost $50 \%$ occasionally or rarely, while $10 \%$ never.

About $30-50 \%$ of cancer patients experience pain and 70-90\% of advanced-stage patients moderate to severe pain [59]. Cancer is the second most common cause of death in Cyprus. However, the cancer death rate in Cyprus is one of the lowest in Europe [35]. The two systematic studies of the use of TENS in patients with cancer pain with 2, n=64 (2008) and 1, n=24 (2012) studies respectively [60] had no significant results due to the small number of studies. In a review of the literature in 2017, although the 9 articles included 
supported the use of TENS for analgesia in cancer patients, the study concluded that there is insufficient evidence to support it [59]. In India, 28\% responded that they use TENS for cancer pain often or very often, and $28 \%$ said they do not use it at all [32]. In Sri Lanka, no physiotherapist replied that uses it in cancer patients very often and $70 \%$ of physiotherapists responded that they did not use it at all [39]. The largest percentage of Cypriots also answered that they do not use TENS (58.4\%) and only $8 \%$ said they use it often or very often. Canser is listed in the diseases that are best avoided by the use of TENS due to unawarness of adverse effects and inadequate literature.This may be one of the reasons for the low use of TENS. Also due to the low rate of cancer patients in Cyprus, compared to the rest of the world, the use of TENS may be limited. From our clinical experience Cypriot patients are not familiar with the use of physiotherapy to reduce cancer pain.

TENS is a method of treatment that has been well studied by the scientific world. Researchers may conclude that the use of TENS is effective in specific groups of patients with specific symptoms, through reviews and meta-analyzes. During their clinical practice, physiotherapists often receive feedback to improve their patients' symptoms and can form a personal clinical opinion. Cypriot physiotherapists believe their patients have little analgesic effect from TENS and only two of the 113 physiotherapists haven't seen any improvement in their patients. The beliefs of Cypriot physiotherapists that their patients benefit from TENS are also reflected in the significant relationship between the effectiveness of TENS and the use frequency to treat pain. Of the $75.3 \%$ of physiotherapists who responded that they use TENS very often or often, $78 \%$ responded that they believed their patients to benefit greatly from the use of TENS and $20.3 \%$ to have little benefit. In India $72 \%$ and $95.5 \%$ of Sri Lankan physiotherapists responded that they believe that their patients benefit greatly from the use of TENS $[32,39]$.

Home treatment reduces the cost that patients have to pay for their medical care. For people with chronic benefit, their medical expenses are high and their coverage in Cyprus, prior to the introduction of the general health system, was mainly payable by the patient as Cyprus offers the lowest medical coverage to its residents (Figure 15) [35]. Due to the low cost purchase, a TENS device can be used at home to reduce the cost of their analgesic treatment. However, TENS is not recommended as a monotherapy and is usually complementary and aids the patient's functional recovery [4]. About $30 \%$ of physiotherapists in Cyprus will not recommend using TENS at home compared to $56 \%$ of Indian physiotherapists and $76.1 \%$ of physiotherapists in Sri Lanka [39]. In UK and Hong Kong, $16.6 \%$ and $50 \%$ physiotherapists respectively believe that TENS will work better when used by the physiotherapist than when the patient himself uses it, while $34.2 \%$ and $8.9 \%$, respectively, believe that TENS will be more effective if used alone at home [52]. TENS when used in the same way by the physiotherapist or the patient will theoretically provide the same effect. However, there is a possibility that the patient may overdose on TENS, resulting in TENS tolerance.

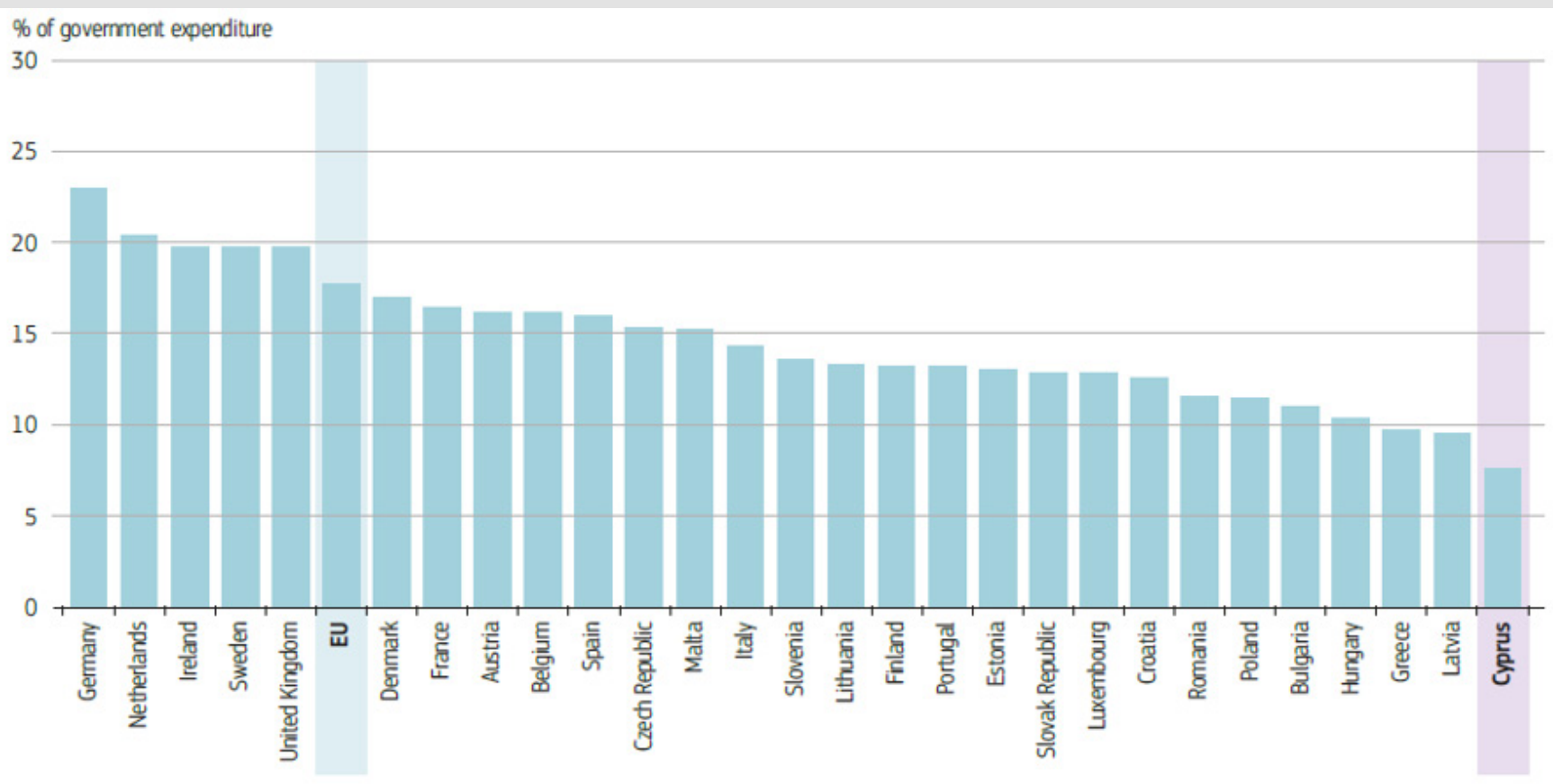

Figure 15: Cyprus spent the smallest amount in Europe for its health system in 2015 [35]. [Vertical axis: Percentage of government expenditure? Horizontal axis: EU Members]. 
In Cyprus, almost 90\% of physiotherapists reported that they had been prescribed by the patient's physician to use TENS for analesia. In India the percentage of physiotherapists recommended by her doctors is about the same (92\%) while in Sri Lanka the percentage is lower by $40.3 \%$ [32,39]. The higher rates in Cyprus and India than in Sri Lanka may be due to better communication between the physician and the physiotherapist.

Patients in Cyprus appear to be familiar with the use of TENS after $71.1 \%$ of physiotherapists said they had been asked for TENS treatment and about half of the physiotherapists responded that they had reported to patients that they had used TENS before receiving treatment for TENS. We believe this question was not fully understood by physiotherapists. The purpose of the question was to determine if the patient had previously received analgesic treatment with TENS, and as we later found could be paraphrased if the patient was treated with TENS just prior to physiotherapy. In India the rates were $68 \%$ and $76 \%$ respectively, and in Sri Lanka $47.7 \%$ and $37.3 \%$ respectively without much difference from Cyprus [32,39].

In the US, two Cohort studies were conducted to investigate the economic efficacy of TENS in patients with chronic low back pain without neurological symptoms. One found that total annual costs were lower in patients who used TENS than in those who did not use $(\mathrm{p}<0.001)$ and in the other, significantly reduced opioid use and lower cost per patient [61,62]. Almost 60\% of Cypriot physiotherapists responded that they believed it to be cost-effective on their part while it is noteworthy that almost 30\% did not know whether it was cost-effective or not. In India 68\% and in Sri Lanka $44.8 \%$ believe that TENS is a cost-effective device for reducing pain $[32,39]$.

The market has a wide range of pain relief devices and machines. There are usually trends in specific ways of analgesia that affect the world, without being informed of their effectiveness by scientific sources. A literature review on the use of electrophysical agents in England, Ireland and Australia (1990-2010) found differences in their availability and use. These differences were mainly identified between countries, depending on whether they were private or public sector and depending on their years of study [38]. In Cyprus 78.7\% physiotherapists have TENS-like devices and provide them often or occasionally, in Sri Lanka 92.2\% and 84\% in India have TENS-like devices TENS [32,39]. Research in US, Israel, Japan, Australia, the UK and Hong Kong on physiotherapists' tendencies to use electrophysiological agents has also shown that physiotherapists have and are using devices similar to TENS [36,37,52,63-64].

The development of research in a small island like Cyprus is very important as our geopolitical position is influenced by different cultures in both health and rehabilitation. It would also be interesting to see how pain is generally treated by Cypriot physiotherapists and how this is affected by private or public sector. It is further suggested that age-related research is needed to observe differences in the physiotherapy practice between younger and older physiotherapists. Due to the small population of Cyprus, large surveys may not be feasible to provide data on the general population, but the island data will be able to be compared with the general population.

\section{Limitations}

The questionnaire was translated not by a qualified translator but by the researcher and the questions were accurately translated. As a result, one of the questiona might not be correctivly understood. This may have distorted the results. The questionnaire also did not specify the age of the attending physiotherapists and their subsequent education. Older physiotherapists have used TENS more and have developed a personal clinical view of its use, effects and effectiveness in practice. In contrast, people who have graduated and get taught about TENS in the recent years have experience of TENS but are likely more updated by newer articles on how to use TENS more effectively. However, there is no suspicion that physiotherapists who have started their practice in recent years do not have a clinical opinion on the use of TENS, nor that older physiotherapists do not have personal and professional development.

\section{Conclusions}

Physiotherapists in Cyprus use of TENS enough and most find it effective. The view of Cypriot physiotherapists is sometimes different and at times similar to other countries. The differences may be due to culture, local education and local clinical practice. The available literature is usually insufficient and does not lead to clear conclusions about how TENS should be used. High quality methodological studies with defined parameter protocols are needed to compare the results with high quality systematic reviews and meta-analyzes to study the efficacy of TENS.

\section{Author Contributions}

The study was conducted for the needs of a postgraduate degree by one of the authors (AP) and supervised by the other (SD).

\section{Funding}

This research received no external funding

\section{Acknowledgments}

We would like to thank all the participants for kindly taking the time to answer our questionnaire.

Conflicts of Interest: The authors declare no conflict of interest.

\section{References}

1. Treede R (2018) The International Association for The Study Of Pain Definition Of Pain. PAIN Reports 3(2): e643.

2. Raffaeli W, Arnaudo E (2017) Pain As A Disease: An Overview. Journal of Pain Research 10: 2003-2008. 
3. Orr P, Shank B, Black A (2017) The Role of Pain Classification Systems in Pain Management. Critical Care Nursing Clinics of North America 29 (4): 407-418

4. Chimenti R, Frey-Law L, Sluka K (2018) A Mechanism-Based Approach to Physical Therapist Management Of Pain. Physical Therapy 98 (5): 302-314.

5. Dubin A, Patapoutian A (2010) Nociceptors: The Sensors of The Pain Pathway. Journal of Clinical Investigation 120(11): 3760-3772.

6. Fricker J (2003) Pain in Europe - A 2003 Report; Cambridge

7. Wiesenfeld-Hallin Z (2005) Sex Differences in Pain Perception. Gender Medicine 2(3): 137-145.

8. Van Hecke O, Torrance N, Smith B (2013) Chronic Pain Epidemiology and Its Clinical Relevance. British Journal of Anaesthesia 111(1): 13-18.

9. Brooks P (2006) The Burden Of Musculoskeletal Disease A Global Perspective. Clinical Rheumatology 25(6): 778-781

10. Birklein F, Dimova V (2017) Complex Regional Pain Syndrome-Up-ToDate. PAIN Reports 2(6): e624.

11. Arendt-Nielsen L, Fernández-de-las-Peñas C, Graven-Nielsen T (2011) Basic Aspects Of Musculoskeletal Pain: From Acute To Chronic Pain. Journal of Manual \& Manipulative Therapy 19(4): 186-193.

12. Mense S (2009) Algesic Agents Exciting Muscle Nociceptors Experimental Brain Research 196(1): 89-100.

13. Woolf C, Ma Q (2007) Nociceptors-Noxious Stimulus Detectors. Neuron 55(3): 353-364.

14. Carr R, Pianova S, McKemy D, Brock J (2009) Action Potential Initiation In The Peripheral Terminals Of Cold-Sensitive Neurones Innervating The Guinea-Pig Cornea. The Journal of Physiology 587 (6): 1249-1264.

15. Heinricher M, Tavares I, Leith J, Lumb B (2009) Descending Control Of Nociception: Specificity, Recruitment And Plasticity. Brain Research Reviews 60(1): 214-225.

16. Jones I, Johnson M (2009) Transcutaneous Electrical Nerve Stimulation. Continuing Education in Anaesthesia Critical Care \& Pain 9 (4): 130-135

17. Vance C, Dailey D, Rakel B, Sluka K (2014) Using TENS For Pain Control: The State Of The Evidence. Pain Management 4(3): 197-209.

18. De Santana J, Walsh D, Vance C, Rakel B, Sluka K (2008) Effectiveness Of Transcutaneous Electrical Nerve Stimulation For Treatment Of Hyperalgesia And Pain. Current Rheumatology Reports 10(6): 492-499.

19. Léonard G, Cloutier C, Marchand S (2011) Reduced Analgesic Effect Of Acupuncture-Like TENS But Not Conventional TENS In Opioid-Treated Patients. The Journal of Pain 12(2): 213-221.

20. Chandra A, Banavaliker J, Das P, Hasti S (2010) Use of Transcutaneous Electrical Nerve Stimulation As An Adjunctive To Epidural Analgesia In The Management of Acute Thoracotomy Pain. Indian Journal of Anaesthesia 54(2): 116-120.

21. Perez Machado A, Santana E, Tacani P, Liebano R (2012) The Effects Of Transcutaneous Electrical Nerve Stimulation On Tissue Repair: A Literature Review. Canadian Journal of Plastic Surgery 20(4): 237-240.

22. Dubinsky R, Miyasaki J (2010) Assessment: Efficacy Of Transcutaneous Electric Nerve Stimulation In The Treatment Of Pain In Neurologic Disorders (An Evidence-Based Review): Report Of The Therapeutics And Technology Assessment Subcommittee of The American Academy Of Neurology. Neurology 74(2): 173-176.

23. Kong X, Gozani S (2018) Effectiveness Of Fixed-Site High-Frequency Transcutaneous Electrical Nerve Stimulation In Chronic Pain: A LargeScale, Observational Study. Journal of Pain Research 11: 703-714.

24. Wolf S, Gersh M, Rao V (1981) Examination Of Electrode Placements And Stimulating Parameters In Treating Chronic Pain With Conventional Transcutaneous Electrical Nerve Stimulation (TENS). Pain 11 (1): 37-47.
25. Mendell L (2014) Constructing And Deconstructing The Gate Theory Of Pain. Pain 155(2): 210-216.

26. Millan M (2002) Descending Control of Pain. Progress in Neurobiology 66(6): 355-474

27. Jamison R, Wan L, Edwards R, Mei A, Ross E (2019) Outcome Of A HighFrequency Transcutaneous Electrical Nerve Stimulator (Hftens) Device For Low Back Pain: A Randomized Controlled Trial. Pain Practice 19(5): 466-475.

28. Ahmed S, Haddad C, Subramaniam S, Khattab S, Kumbhare D (2019) The Effect Of Electric Stimulation Techniques On Pain And Tenderness At The Myofascial Trigger Point: A Systematic Review. Pain Medicine 20(9): 1774-1788.

29. Dailey D, Rakel B, Vance C, Liebano R, Amrit A etal. (2013) Transcutaneous Electrical Nerve Stimulation Reduces Pain, Fatigue And Hyperalgesia While Restoring Central Inhibition In Primary Fibromyalgia. Pain, 154(11): 2554-2562.

30. Bergeron-Vézina K, Corriveau H, Martel M, Harvey M, Léonard G (2015) High- And Low-Frequency Transcutaneous Electrical Nerve Stimulation Does Not Reduce Experimental Pain In Elderly Individuals. PAIN 156(10): 2093-2099.

31. Butera K, George S, Borsa P, Dover G (2018) Prolonged Reduction In Shoulder Strength After Transcutaneous Electrical Nerve Stimulation Treatment of Exercise-Induced Acute Muscle Pain. Pain Practice 18 (8): 954-968.

32. Gourav Banerjee, Mark I Johnson (2013) A Survey of Physiotherapist's attitudes and beliefs about the use of TENS for pain management in India. International Journal of Scientific Research and Reviews 2(3): 3646.

33. Egan M, Seeger D, Schöps P (2015) Physiotherapie Und Physikalische Therapie In Der Schmerzmedizin. Der Schmerz 29(5): 562-568.

34. Semmons J (2016) The Role Of Physiotherapy In The Management Of Chronic Pain. Anaesthesia \& Intensive Care Medicine 17 (9): 445-447.

35. (2017) Cyprus: Country Health Profile 2017. State of Health in the EU 2017

36. Chipchase L, Williams M, Robertson V (2009) A National Study Of The Availability And Use Of Electrophysical Agents By Australian Physiotherapists. Physiotherapy Theory and Practice 25(4): 279-296.

37. Springer S, Laufer Y, Elboim-Gabyzon M (2015) Clinical Decision Making For Using Electro-Physical Agents By Physiotherapists, An Israeli Survey. Israel Journal of Health Policy Research 4(1).

38. Shah S, Farrow A (2012) Trends In The Availability And Usage Of Electrophysical Agents In Physiotherapy Practices From 1990 To 2010: A Review. Physical Therapy Reviews 17(4): 207-226.

39. Dissanayakae T, Banerjee G, Johnson M (2014) A Survey Of The Attitudes And Beliefs About The Use Of TENS For Pain Management By Physiotherapists Working In Two Cities In Sri Lanka. Patient Related Outcome Measures 5: 35-41.

40. Reeves J, Graff-Radford S, Shipman D (2004) The Effects Of Transcutaneous Electrical Nerve Stimulation On Experimental Pain And Sympathetic Nervous System Response. Pain Medicine 5(2): 150-161.

41. Nnoaham K, Kumbang J (2008) Transcutaneous Electrical Nerve Stimulation (TENS) For Chronic Pain. Cochrane Database of Systematic Reviews 16(3).

42. Johnson M, Claydon L, Herbison G, Jones G, Paley C (2017) Transcutaneous Electrical Nerve Stimulation (TENS) For Fibromyalgia In Adults. Cochrane Database of Systematic Reviews.

43. Henschke N, Kamper S, Maher C (2015) The Epidemiology And Economic Consequences Of Pain. Mayo Clinic Proceedings 90(1): 139-147.

44. Linton S, Shaw W (2011) Impact of Psychological Factors In The Experience Of Pain. Physical Therapy 91(5): 700-711. 
45. Smith B, Hopton J, Chambers W (1999) Chronic pain in primary care. Family Practice 16(5): 475-482.

46. Gardner S, Blodgett N, Hillis S, Borhart E, Malloy L, et al. (2014) HI-TENS Reduces Moderate-To-Severe Pain Associated With Most Wound Care Procedures. Biological Research For Nursing 16(3): 310-319.

47. Milne S, Welch V, Brosseau L, Saginur M, Shea B, et al. (2001) Transcutaneous electrical nerve stimulation (TENS) for chronic lowback pain (Review). Cochrane Database of Systematic Reviews (2).

48. Sluka K (2002) Stimulation Of Deep Somatic Tissue With Capsaicin Produces Long-Lasting Mechanical Allodynia And Heat Hypoalgesia That Depends On Early Activation Of The Camp Pathway. The Journal of Neuroscience 22(13): 5687-5693.

49. Vance C, Rakel B, Blodgett N, DeSantana J, Amendola A, et al. (2012) Effects of Transcutaneous Electrical Nerve Stimulation On Pain, Pain Sensitivity, And Function In People With Knee Osteoarthritis: A Randomized Controlled Trial. Physical Therapy 92(7): 898-910.

50. Johnson M, Martinson M (2007) Efficacy of Electrical Nerve Stimulation For Chronic Musculoskeletal Pain: A Meta-Analysis Of Randomized Controlled Trials. Pain 130(1): 157-165.

51. Gibson W, Wand B, O'Connell N (2017) Transcutaneous Electrical Nerve Stimulation (TENS) For Neuropathic Pain In Adults. Cochrane Database of Systematic Reviews (9).

52. Scudds R, Scudds R, Baxter G, McDonough S, Walsh D (2009) Transcutaneous Electrical Nerve Stimulation For The Treatment Of Pain In Physiotherapy Practices In Hong Kong And The United Kingdom-A Survey Of Usage And Perceived Effectiveness Compared With Other Pain Relieving Modalities. Hong Kong Physiotherapy Journal 27(1): 11-20.

53. Mahmood A, Veluswamy S, Hombali A, Mullick A, N M, Solomon J (2019) Effect Of Transcutaneous Electrical Nerve Stimulation On Spasticity In Adults With Stroke: A Systematic Review And Meta-Analysis. Archives of Physical Medicine and Rehabilitation 100(4): 751-768.

54. Carroll D, Tramèr M, McQuay H, Nye B, Moore A (1996) Randomization Is Important In Studies With Pain Outcomes: Systematic Review Of Transcutaneous Electrical Nerve Stimulation In Acute Postoperative Pain. British Journal of Anaesthesia 77(6): 798-803.

55. Zhu Y, Feng Y, Peng L (2017) Effect Of Transcutaneous Electrical Nerve Stimulation For Pain Control After Total Knee Arthroplasty: A Systematic

ISSN: 2574-1241

DOI: 10.26717/BJSTR.2019.23.003948

Dimitrios Stasinopoulos. Biomed J Sci \& Tech Res

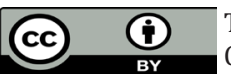

This work is licensed under Creative

Commons Attribution 4.0 License

Submission Link: https://biomedres.us/submit-manuscript.php
Review And Meta-Analysis. Journal of Rehabilitation Medicine 49 (9): 700-704.

56. Bjordal J, Johnson M, Ljunggreen A (2003) Transcutaneous Electrical Nerve Stimulation (TENS) Can Reduce Postoperative Analgesic Consumption. A Meta-Analysis With Assessment of Optimal Treatment Parameters For Postoperative Pain. European Journal of Pain 7(2): 181188.

57. Hurlow A, Bennett M, Robb K, Johnson M, Simpson K, et al. (2008) Transcutaneous electric nerve stimulation (TENS) for cancer pain in adults. Cochrane Database of Systematic Reviews (3).

58. Hurlow A, Bennett M, Robb K, Johnson M, Simpson K et al. (2012) Transcutaneous electric nerve stimulation (TENS) for cancer pain in adults (Review). Cochrane Database of Systematic Reviews (3).

59. Salim N, Nigim H (2017) Effect Of Transcutaneous Electrical Nerve Stimulation (TENS) On Pain Among Patients With Cancer. Advanced Practices in Nursing 2(2).

60. Bae Y, Lee S (2014) Analgesic Effects of Transcutaneous Electrical Nerve Stimulation And Interferential Current On Experimental Ischemic Pain Models: Frequencies of $50 \mathrm{~Hz}$ And $100 \mathrm{~Hz}$. Journal of Physical Therapy Science 26(12): 1945-1948.

61. Pivec R, Stokes M, Chitnis A, Paulino C, Harwin S, et al. (2013) Clinical And Economic Impact Of TENS In Patients With Chronic Low Back Pain: Analysis of A Nationwide Database. Orthopedics 36(12): 922-928.

62. Pivec R, Minshall M, Mistry J, Chughtai M, Elmallah R, et al. (2015) Decreased Opioid Utilization and Cost at One Year in Chronic Low Back Pain Patients Treated with Transcutaneous Electric Nerve Stimulation (TENS). Surgical technology international 27: 268-274.

63. Greco J, Lamberg E, McKenna R, Muratori L (2018) Trends in Availability And Usage Of Biophysical Agents Among Physical Therapists In The United States. Physical Therapy Reviews 23(2): 116-123.

64. Abe Y, Goh A, Miyoshi K (2016) Availability, Usage, And Factors Affecting Usage Of Electrophysical Agents By Physical Therapists: A Regional Cross-Sectional Survey. Journal of Physical Therapy Science 28 (11): 3088-3094.

65. de Lalouvière L, Ioannou Y, Fitzgerald M (2014) Neural Mechanisms Underlying The Pain Of Juvenile Idiopathic Arthritis. Nature Reviews Rheumatology 10(4): 205-211.

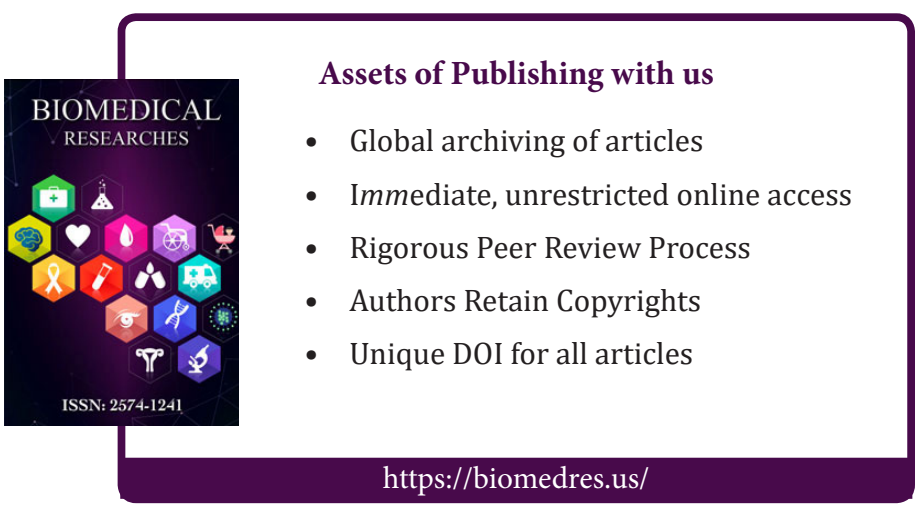

Copyright@ Dimitrios Stasinopoulos | Biomed J Sci \& Tech Res | BJSTR. MS.ID.003948. 